IOS Press

\title{
Thesis
}

\section{Ontologies for interaction: Enabling serendipitous interoperability in smart environments}

\author{
Gerrit Niezen \\ Department of Industrial Design, Eindhoven University of Technology, Eindhoven, The Netherlands \\ Currently with: Swansea University, Wales, United Kingdom \\ E-mail: gniezen@ieee.org
}

Keywords: Ontologies, semantic web, software architecture, interaction design

On 9 October 2012 Gerrit Niezen successfully defended his PhD dissertation at Eindhoven University of Technology, the Netherlands, entitled: "Ontologies for interaction: Enabling serendipitous interoperability in smart environments". The work was supported by the SOFIA (Smart Objects For Intelligent Applications) project and funded through European JTI ARTEMIS under the subprogramme "Smart Environments and Scalable Digital Services".

Gerrit defended his dissertation in a public ceremony held at Eindhoven University of Technology. The thesis was supervised by Prof. Loe Feijs and Prof. Panos Markopoulos, and co-supervised by Dr. Jun Hu. The members of the reading committee included Prof. Harold Thimbleby, Dr. Stephan Wensveen and Prof. Johan Lukkien.

\section{Thesis summary}

The thesis describes the design and development of an ontology and software framework to support user interaction in ubiquitous computing scenarios. The key goal of ubiquitous computing is "serendipitous interoperability", where devices that were not necessarily designed to work together should be able to discover each other's functionality and be able to make use of it. Future ubiquitous computing scenarios involve hundreds of devices. Therefore, anticipating all the different types of devices and usage scenarios a priori is an unmanageable task.

An iterative approach was followed during the design process, with three design iterations documented in the thesis. The work was completed in close collaboration with another $\mathrm{PhD}$ candidate, Bram van der Vlist, whose thesis [4] describes the more designer-related aspects in greater detail. The work was also done in close cooperation with the other project partners, in order to elicit requirements and maintain a more holistic view of the various application areas.

The thesis describes an interaction model that shows the various concepts that are involved in user interaction in a smart space, including how these concepts work together. Based in the interaction model, a theory of semantic connections is introduced that focuses on the meaning of the connections between the different entities in a smart environment [5].

Ontologies are formal representations of concepts in a domain of interest and the relationships between these concepts. They are used to enable the exchange 


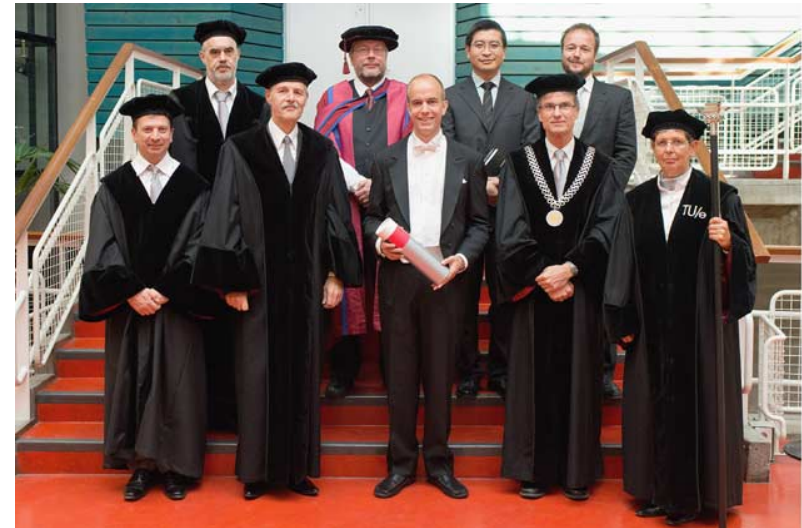

of information without requiring up-front standardisation. The ontology described in the thesis helps developers to focus on modelling the interaction capabilities of smart objects [6] and inferring the possible connections between these objects, making it easier to build smart objects and enable device interoperability on a semantic level [3].

The ontology and software architecture described in the thesis has proven to be easier to use and more flexible than existing methods, like storing device and service descriptions in a relational database. Developers were able to make use of existing ontology concepts as defined, as well as define their own concepts where necessary. Once familiar with how ontologies work, they found the ontology easy to understand. Ontologies also made the relationships between various entities more visible.

Rather than just describing the low-level hardware input event that triggered an action, interaction events in the ontology are modelled as high-level input actions which report the intent of the user's action directly. This allows developers to write software that respond to these high-level events, without having to support every kind of device that could have generated that event. The event hierarchy can be inferred using semantic reasoning.

The software architecture implements the publish/subscribe messaging paradigm, enabling smart objects to subscribe to changes in data, represented in triple form, and be notified every time these triples are updated, added or removed. Semantic reasoning is performed on an information broker, simplifying the implementation on the smart objects.

A pilot deployment, composed of heterogeneous smart objects designed and manufactured by a range of companies and institutions, was used to validate the design. A performance evaluation was performed

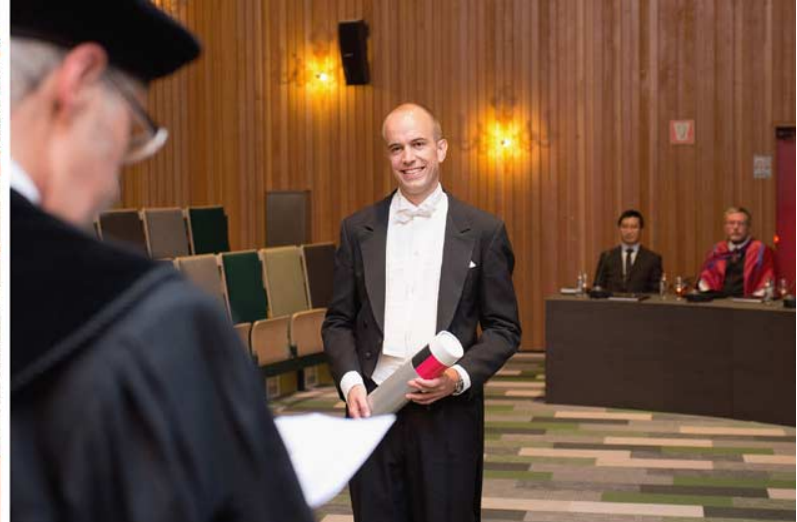

[2], where the results indicated acceptable response times for a networked user interface. The software architecture compares favourably to systems like the EventHeap [1], that also used a tuple space protocol to establish communication between devices. EventHeap was shown to provide latency below $100 \mathrm{~ms}$ for 12 different applications each generating and receiving 10 events per second. The query time measurements for the architecture described in the thesis were also all below $100 \mathrm{~ms}$. Although the subscription measurements described in the thesis were slower, the time required for reasoning improves the flexibility and capabilities of the system to such an extent that it is worth the hit in performance.

A usability analysis of the ontology and system implementation was performed using a developer questionnaire based on an existing usability framework. Various ontology design patterns were identified during the course of the design, and are documented in the thesis.

The resulting design artefact is an ontology for user interaction with devices in a smart environment, where devices are able to share interaction events and make use of each other's functionality.

\section{References}

[1] B. Johanson and A. Fox, The Event Heap: A coordination infrastructure for interactive workspaces, in: Proc. Fourth IEEE Workshop on Mobile Computing Systems and Applications, 2002, pp. 83-93.

[2] G. Niezen, B.J.J. van der Vlist, S. Bhardwaj, and T. Ozcelebi, Performance evaluation of a semantic smart space deployment, in: 4th International Workshop on Sensor Networks and Ambient Intelligence (SeNAmI 2012), Lugano, Switzerland, 2012.

[3] G. Niezen, B.J.J. van der Vlist, J. Hu, and L.M.G. Feijs, Using semantic transformers to enable interoperability between media devices in a ubiquitous computing environment, in: Grid 
and Pervasive Computing Workshops, Lecture Notes in Computer Science, Vol. 7096, Springer, Berlin/Heidelberg, 2011, pp. 44-53.

[4] B.J.J. van der Vlist, Designing semantic connections: Explorations, theory and a framework for design, $\mathrm{PhD}$ thesis, Eindhoven University of Technology, 2012.
[5] B.J.J. van der Vlist, J. Hu, G. Niezen, and L.M.G. Feijs, Design semantics of connections of a smart home environment, Creation and Design, 13(02) (2012), 18-24.

[6] B.J.J. van der Vlist, G. Niezen, J. Hu, and L.M.G. Feijs, Interaction primitives: Describing interaction capabilities of smart objects in ubiquitous computing environments, in: IEEE Africon 2011, Livingstone, Zambia, 2011. 